\title{
Sequent Calculi for the classical fragment of Bochvar and Halldén's Nonsense Logics
}

\author{
Marcelo E. Coniglio María I. Corbalán \\ Centre for Logic, Epistemology and the History of Science \\ and \\ Department of Philosophy \\ State University of Campinas, Brazil \\ coniglio@cle.unicamp.br inescorbalan@yahoo.com.ar
}

\begin{abstract}
In this paper sequent calculi for the classical fragment (that is, the conjunction-disjunction-implication-negation fragment) of the nonsense logics $\mathbf{B}_{3}$, introduced by Bochvar, and $\mathbf{H}_{3}$, introduced by Halldén, are presented. These calculi are obtained by restricting in an appropriate way the application of the rules of a sequent calculus for classical propositional logic CPL. The nice symmetry between the provisos in the rules reveal the semantical relationship between these logics. The Soundness and Completeness theorems for both calculi are obtained, as well as the respective Cut elimination theorems.
\end{abstract}

\section{Introduction}

The study of logical paradoxes from a formal perspective has produced several proposals in the literature. In particular, 3-valued propositional logics were proposed in which, besides the two 'classical' truth-values, the third one plays the role of a 'nonsensical' or 'meaningless' truth value. This is why these logics are known as 'logics of nonsense'. In 1938 ([3]) A. Bochvar introduced the first logic of nonsense, by means of 3-valued logical matrices. Since the nonsensical truth value is not distinguished, Bochvar's logic is paracomplete but it is not paraconsistent: the negation $\neg$ is explosive (from a contradiction everything follows) but the third-excluded law does not hold. In 1949 S. Halldén ([6]) proposed a closely related logic of nonsense by means of 3-valued logical matrices in which the third truth-value is distinguished, producing a paraconsistent, non-paracomplete logic.

Both logics share the same main feature: the nonsensical truth-value is 'infectious' in the sense that, given a valuation $v$, every formula having at least one propositional variable with nonsensical truth-value under $v$ also gets the non-sensical truth-value under $v$. Also, both logics contain, besides the connectives $\neg$ for negation and $\wedge$ conjunction, an unary connective which allows to recover all the classical inferences (cf. [4, 5]).

The respective 'classical' fragments of each of these two logics (that is, the $\{\neg, \vee, \wedge, \rightarrow\}$-fragments) are interesting since they together constitute the only two possibilities for extending the usual matrices of classical logic with a third nonsensical, 'infectious' truth-value $\frac{1}{2}$ : either $\frac{1}{2}$ is designated or it is not. The former corresponds to the 'classical' fragment of Halldén's logic, while the latter corresponds to the same fragment of Bochvar's logic. It is not hard to establish, by semantical means, a relationship between these two fragments and classical logic: given a classically valid inference $\Gamma \vdash \alpha$ over the language generated by $\{\neg, \vee, \wedge, \rightarrow\}$, if the propositional variables ocurring in $\Gamma$ also occur in $\alpha$ then $\Gamma \vdash \alpha$ is valid in Halldén's logic $\mathbf{H}_{3}$. Dually, if the propositional variables ocurring in $\alpha$ also occur in $\Gamma$ then such classically valid inference is valid in Bochvar's logic $\mathbf{B}_{3}$. This duality is a direct consequence

D. Kesner and P. Viana (Eds.): LSFA 2012

EPTCS 113, 2013, pp. 125136 doi 10.4204/EPTCS.113.12 (c) M. E. Coniglio \& M. I. Corbalán

This work is licensed under the Creative Commons Attribution License. 
of the criterion adopted in each logic with respect to the third truth-value (namely, designated vs. nondesignated), and the fact that this non-sensical truth-value propagates through any complex formula. Since $\{\vee, \rightarrow\}$ and $\{\wedge, \rightarrow\}$ can be defined as usual from $\{\neg, \wedge\}$ and $\{\neg, \vee\}$, respectively, the observation above can also be applied to the $\{\neg, \wedge\}$ and $\{\neg, \vee\}$-fagments of both logics.

This paper introduced two cut-free sequent calculi for the $\{\neg, \vee, \wedge, \rightarrow\}$-fragment of each logic of nonsense mentioned above. Both systems are obtained by imposing restrictions on the rules of the usual sequent calculus for classical propositional logic CPL. In the calculus for the classical fragment of Halldén's logic, the introduction rules for conjunction, implication and negation on the left side of the sequent are restricted. In the calculus for the fragment of Bochvar's logic the restriction is imposed to the introduction rule for disjunction, implication and negation on the right side. In this manner, the relationship between classical logic and both logics became explicit through restrictions on the rules for the logical connectives $\neg, \wedge, \vee$ and $\rightarrow$.

\section{Preliminaries}

Along this paper, we fix a denumerable set prop of propositional variables, as well as three propositional signatures: $\Sigma_{1}$ just containing a negation (unary) connective $\neg$ and a disjunction (binary) connective $\vee$; $\Sigma_{2}$ just containing negation $\neg$ and a conjunction (binary) connective $\wedge$; and $\Sigma_{0}$, containing $\neg, \vee, \wedge$, and an implication (binary) connective $\rightarrow$. The set of formulas generated by $\Sigma_{i}$ and prop will be denoted by For $_{i}$, for $i=0,1,2$. The disjunction $\vee$ and the implication $\rightarrow$ are defined in For 2 as $\alpha \vee \beta=$ def $\neg(\neg \alpha \wedge \neg \beta)$ and $\alpha \rightarrow \beta=$ def $\neg(\alpha \wedge \neg \beta)$, respectively. By its turn, the conjunction $\wedge$ and the implication $\rightarrow$ are defined in For $_{1}$ as $\alpha \wedge \beta={ }_{\text {def }} \neg(\neg \alpha \vee \neg \beta)$ and $\alpha \rightarrow \beta={ }_{\text {def }} \neg \alpha \vee \beta$, respectively.

For $i=0,1,2$, the function var : For $\rightarrow \wp($ prop $)$ which assigns to each formula the set of propositional variables appearing in it is defined recursively as usual. When $\Gamma \subseteq F_{o r}$ is a set of formulas then $\operatorname{var}(\Gamma)=\bigcup_{\gamma \in \Gamma} \operatorname{var}(\gamma)$.

The next step is to recall a well-known cut-free sequent calculus for classical propositional logic CPL defined over the signature $\Sigma_{0}$.

Definition 1 By a sequent $\mathrm{S}$ over $\Sigma_{i}(i=0,1,2)$ we shall mean an ordered pair $\langle\Gamma, \Delta\rangle$ of (non-simultaneously empty) finite sets of formulas in For . $_{\text {. }}$

We shall use the more suggestive notation $\Gamma \Rightarrow \Delta$ for the sequent $\langle\Gamma, \Delta\rangle$. Sequents of the form $\langle\Gamma, \emptyset\rangle$, $\langle\emptyset, \Delta\rangle,\langle\Gamma,\{\alpha\}\rangle$ and $\langle\{\alpha\}, \Delta\rangle$ will be denoted by $\Gamma \Rightarrow, \Rightarrow \Delta, \Gamma \Rightarrow \alpha$ and $\alpha \Rightarrow \Delta$, respectively. As usual, we write $\alpha, \Gamma$ (or $\Gamma, \alpha$ ) and $\alpha, \beta, \Gamma$ (or $\Gamma, \alpha, \beta$ ) instead of $\Gamma \cup\{\alpha\}$ and $\Gamma \cup\{\alpha, \beta\}$, respectively.

Definition 2 The sequent calculus $\boldsymbol{C}$ over $\Sigma_{0}$ is defined as follows:

\section{Axioms}

$$
A x \overline{\alpha \Rightarrow \alpha}
$$

\section{Structural rules}

$$
W \Rightarrow \frac{\Gamma \Rightarrow \Delta}{\alpha, \Gamma \Rightarrow \Delta} \quad \Rightarrow W \frac{\Gamma \Rightarrow \Delta}{\Gamma \Rightarrow \Delta, \alpha} \quad C u t \frac{\Gamma \Rightarrow \Delta, \alpha \quad \alpha, \Gamma \Rightarrow \Delta}{\Gamma \Rightarrow \Delta}
$$




\section{Operational rules}

$$
\begin{gathered}
\neg \Rightarrow \frac{\Gamma \Rightarrow \Delta, \alpha}{\neg \alpha, \Gamma \Rightarrow \Delta} \quad \Rightarrow \neg \frac{\alpha, \Gamma \Rightarrow \Delta}{\Gamma \Rightarrow \Delta, \neg \alpha} \\
\wedge \Rightarrow \frac{\alpha_{1}, \alpha_{2}, \Gamma \Rightarrow \Delta}{\alpha_{1} \wedge \alpha_{2}, \Gamma \Rightarrow \Delta} \quad \Rightarrow \wedge \frac{\Gamma \Rightarrow \Delta, \alpha_{1} \quad \Gamma \Rightarrow \Delta, \alpha_{2}}{\Gamma \Rightarrow \Delta, \alpha_{1} \wedge \alpha_{2}} \\
\vee \Rightarrow \frac{\alpha_{1}, \Gamma \Rightarrow \Delta \quad \alpha_{2}, \Gamma \Rightarrow \Delta}{\alpha_{1} \vee \alpha_{2}, \Gamma \Rightarrow \Delta} \\
\rightarrow \frac{\Gamma \Rightarrow \Delta, \alpha_{1} \quad \alpha_{2}, \Gamma \Rightarrow \Delta}{\alpha_{1} \rightarrow \alpha_{2}, \Gamma \Rightarrow \Delta} \quad \Rightarrow \vee \frac{\Gamma \Rightarrow \Delta, \alpha_{1}, \alpha_{2}}{\Gamma \Rightarrow \Delta, \alpha_{1} \vee \alpha_{2}} \\
\Rightarrow \rightarrow \frac{\alpha_{1}, \Gamma \Rightarrow \Delta, \alpha_{2}}{\Gamma \Rightarrow \Delta, \alpha_{1} \rightarrow \alpha_{2}}
\end{gathered}
$$

For $i=0,1,2$, consider the usual classical valuations from For $_{i}$ over the set $V_{\mathbf{C P L}}=\{1,0\}$ of classical truth-values, where 1 denotes the "true" value and 0 denotes the "false" value. Let $\vDash_{\text {CPL }}$ be the semantical consequence relation of CPL over For 0 , that is: $\Gamma \vDash_{\mathbf{C P L}} \alpha$ iff, for every classical valuation $v$ : if $v(\gamma)=1$ for every $\gamma \in \Gamma$ then $v(\alpha)=1$. The following theorems are well known:

Theorem 3 (Soundness and Completeness of $\mathbf{C}$ ) Let $\Gamma \cup \Delta$ be a finite set of formulas in For 0 . Then: the sequent $\Gamma \Rightarrow \Delta$ is provable in $C$ iff $\Gamma \vDash_{\mathbf{C P L}} \bigvee_{\alpha \in \Delta} \alpha .1$ In particular: the sequent $\Gamma \Rightarrow \alpha$ is provable in $\boldsymbol{C}$ iff $\Gamma \vDash_{\mathbf{C P L}} \alpha$. The same holds for the $\{\neg, \vee\}$ and the $\{\neg, \wedge\}$-fragments of $\boldsymbol{C}$.

Theorem 4 (Cut elimination for C) Let $\Gamma \cup \Delta$ be a finite nonempty set of formulas in For 0 . If the sequent $\Gamma \Rightarrow \Delta$ is provable in $\boldsymbol{C}$ then there is a cut-free derivation of it in $\boldsymbol{C}$, that is, a derivation without using the Cut rule. The same holds for the $\{\neg, \vee\}$ and the $\{\neg, \wedge\}$-fragments of $\boldsymbol{C}$.

\section{Three-valued nonsense logics $\mathrm{B}_{3}$ and $\mathrm{H}_{3}$}

The logics of nonsense $\mathbf{B}_{3}$ of Bochvar and $\mathbf{H}_{3}$ of Halldén are three-valued logics. Their set of truth-values is $V=\left\{1, \frac{1}{2}, 0\right\}$ where the third non-classical truth-value $\frac{1}{2}$ is interpreted as a nonsensical truth-value. In $\mathbf{H}_{3}$ this third truth-value is designated; on the other hand, $\frac{1}{2}$ is undesignated in $\mathbf{B}_{3}$. So, $D_{\mathbf{B}_{3}}=\{1\}$ is the set of designated values of $\mathbf{B}_{3}$ and $D_{\mathbf{H}_{3}}=\left\{1, \frac{1}{2}\right\}$ is the set of designated values of Halldén's logic $\mathbf{H}_{3}$. The logic $\mathbf{B}_{3}$ is defined over the signature $\Sigma_{2}^{\#_{\mathbf{B}}}$ obtained from the signature $\Sigma_{2}$ by adding an unary 'meaningful' connective $\#_{\mathbf{B}}$. By its turn, $\mathbf{H}_{3}$ is defined over the signature $\Sigma_{1}^{\#_{\mathbf{H}}}$ obtained from $\Sigma_{1}$ by adding an unary 'meaningful' connective $\#_{\mathbf{H}}$. By means of the connectives $\#_{\mathbf{B}}$ and $\#_{\mathbf{H}}$ it is possible to express the meaninglessness of a formula at the object-language level of each logic. The abbreviations for defining the other classical connectives in each signature are the same as in CPL (recall Section 1). The truthtables for negation, conjunction, disjunction, implication and meaningful connectives in $\mathbf{B}_{3}$ and $\mathbf{H}_{3}$ are as follows:

\begin{tabular}{l|l} 
& $\neg$ \\
\hline 1 & 0 \\
$\frac{1}{2}$ & $\frac{1}{2}$ \\
0 & 1
\end{tabular}

\begin{tabular}{c|ccc}
$\wedge$ & 1 & $\frac{1}{2}$ & 0 \\
\hline 1 & 1 & $\frac{1}{2}$ & 0 \\
$\frac{1}{2}$ & $\frac{1}{2}$ & $\frac{1}{2}$ & $\frac{1}{2}$ \\
0 & 0 & $\frac{1}{2}$ & 0
\end{tabular}

\begin{tabular}{c|ccc}
$\vee$ & 1 & $\frac{1}{2}$ & 0 \\
\hline 1 & 1 & $\frac{1}{2}$ & 1 \\
$\frac{1}{2}$ & $\frac{1}{2}$ & $\frac{1}{2}$ & $\frac{1}{2}$ \\
0 & 1 & $\frac{1}{2}$ & 0
\end{tabular}

\begin{tabular}{c|ccc}
$\rightarrow$ & 1 & $\frac{1}{2}$ & 0 \\
\hline 1 & 1 & $\frac{1}{2}$ & 0 \\
$\frac{1}{2}$ & $\frac{1}{2}$ & $\frac{1}{2}$ & $\frac{1}{2}$ \\
0 & 1 & $\frac{1}{2}$ & 1
\end{tabular}

\footnotetext{
${ }^{1}$ Here, $\bigvee_{\alpha \in \Delta} \alpha$ denotes the formula $\alpha_{1} \vee\left(\alpha_{2} \vee \cdots\left(\alpha_{n-1} \vee \alpha_{n}\right) \ldots\right)$, if $\Delta=\left\{\alpha_{1}, \ldots, \alpha_{n}\right\}$. If $\Delta=\{\alpha\}$ or $\Delta=\emptyset$ then $\bigvee_{\alpha \in \Delta} \alpha=$ $\alpha$ and $\bigvee_{\alpha \in \Delta} \alpha=p_{1} \wedge \neg p_{1}$, respectively, where $p_{1}$ is the first propositional variable.
} 


\begin{tabular}{c|ccc|c} 
& $\#_{\mathbf{B}}$ & & $\#_{\mathbf{H}}$ \\
\hline 1 & 1 & & 1 & 1 \\
$\frac{1}{2}$ & 0 & & $\frac{1}{2}$ & 0 \\
0 & 0 & & 0 & 1
\end{tabular}

Additionally, $\#_{\mathbf{H}}$ can be defined in terms of the connectives of $\mathbf{B}_{3}$ but the same relationship between $\#_{\mathbf{B}}$ and $\mathbf{H}_{3}$ is not true, and so the expressive power of the matrices of $\mathbf{B}_{3}$ is strictly stronger than that of the matrices of $\mathbf{H}_{3}$ (cf. [5]).

The key feature of both logics is the following, which can be easily proved by induction on the complexity of the formula $\alpha$ :

Proposition 5 Let $\alpha$ be a formula of $\boldsymbol{L}$ without \# and let $v$ be a valuation of $\boldsymbol{L}$, where $\boldsymbol{L}=\boldsymbol{B}_{3}$ or $\boldsymbol{L}=\boldsymbol{H}_{3}$. Then: $v(\alpha)=\frac{1}{2}$ iff $v(p)=\frac{1}{2}$ for some propositional variable $p \in \operatorname{var}(\alpha)$.

This means that in the 'classical' fragment of $\mathbf{B}_{3}$ and $\mathbf{H}_{3}$ the non-classical truth-value $\frac{1}{2}$ is 'infectious': an atomic formula 'infects' complex formulas with the nonsensical truth-value. It is easy to prove that, over the respective $\Sigma_{i}$, both $\mathbf{B}_{3}$ and $\mathbf{H}_{3}$ are deductive fragments of classical logic: every valid inference in $\mathbf{B}_{3}$ or in $\mathbf{H}_{3}$ written in the classical signature $\Sigma_{i}$ is valid in CPL. In fact, the following proposition (whose proof is immediate) holds in $\mathbf{B}_{3}$ and $\mathbf{H}_{3}$.

Proposition 6 Let $\alpha$ be a formula of $\boldsymbol{L}$ without \#, let $v_{\mathbf{C P L}}$ be a classical valuation and let $v_{\mathbf{L}}$ be a valuation of $\boldsymbol{L}$, where $\boldsymbol{L}=\boldsymbol{B}_{3}$ or $\boldsymbol{L}=\boldsymbol{H}_{3}$. If $v_{\mathbf{L}}(p)=v_{\mathbf{C P L}}(p)$ for every propositional variable $p \in \operatorname{var}(\alpha)$ then $v_{\mathbf{L}}(\alpha)=v_{\mathbf{C P L}}(\alpha)$ (and so $v_{\mathbf{L}}(\alpha) \in\{1,0\}$ ).

Despite these similarities, there are important differences between $\mathbf{B}_{3}$ and $\mathbf{H}_{3}$ with respect to classical logic as a consequence of choosing different sets of designed truth-valued:

- There are no tautological formulas over $\Sigma_{2}$ in $\mathbf{B}_{3} ; \mathbf{H}_{3}$ contain every classical tautology over $\Sigma_{1}$.

- No contradiction written over $\Sigma_{1}$ is a trivializing formula in $\mathbf{H}_{3}$; every contradiction over $\Sigma_{2}$ is a trivializing formula in $\mathbf{B}_{3}$.

- The Deduction Theorem is not valid in $\mathbf{B}_{3}$ and modus ponens is not valid in $\mathbf{H}_{3}$. So, the following metaproperty does not hold in $\mathbf{B}_{3}$ : if $\Gamma, \alpha \vDash \beta$, then $\Gamma \vDash \alpha \rightarrow \beta$; on the other hand, the following metaproperty does not hold in $\mathbf{H}_{3}$ : if $\Gamma \vDash \alpha \rightarrow \beta$, then $\Gamma, \alpha \vDash \beta$.

- The inference $\alpha \vDash \alpha \vee \beta$ does not hold in $\mathbf{B}_{3}$; in $\mathbf{H}_{3}$ the inference $\alpha \wedge \beta \vDash \alpha$ does not hold.

- In $\mathbf{B}_{3}$ the Principle of Excluded Middle:

$$
\vDash \alpha \vee \neg \alpha
$$

does not hold; in $\mathbf{H}_{3}$ the Principle of Explosion:

$$
\alpha, \neg \alpha \vDash \beta
$$

does not hold. Thus, $\mathbf{B}_{3}$ is paracomplete w.r.t. the negation $\neg$, while $\mathbf{H}_{3}$ is paraconsistent w.r.t. $\neg$.

These differences between Bochvar and Halldén's connectives with respect to classical connectives are not independent from each other, and their connections are expressed in the following theorems, which constitute the basis of our proposal.

Theorem 7 Let $\Gamma \cup\{\alpha\}$ be a set of formulas in For 2 such that $\Gamma \vDash_{\mathbf{C P L}} \alpha$. Then:

$$
\text { if } \operatorname{var}(\alpha) \subseteq \operatorname{var}(\Gamma) \text { or } \Gamma \vDash_{\mathbf{C P L}} p_{1} \wedge \neg p_{1} \text { then } \Gamma \vDash_{\mathbf{B}_{3}} \alpha \text {. }
$$


Proof. Cf. [3, 9, 7, 5].

Theorem 8 Let $\Gamma \cup\{\alpha\}$ be a set of formulas in For 1 such that $\Gamma \vDash_{\mathbf{C P L}} \alpha$. Then:

$$
\text { if } \operatorname{var}(\Gamma) \subseteq \operatorname{var}(\alpha) \text { or } \vDash_{\mathbf{C P L}} \alpha \text { then } \Gamma \vDash_{\mathbf{H}_{3}} \alpha \text {. }
$$

Proof. Assume that $\Gamma \vDash_{\mathbf{C P L}} \alpha$. If $\Gamma \nvdash_{\mathbf{H}_{3}} \alpha$ then there is a valuation $v_{\mathbf{H}_{3}}$ for $\mathbf{H}_{3}$ such that $v_{\mathbf{H}_{3}}(\Gamma) \subseteq$ $\left\{1, \frac{1}{2}\right\}$ and $v_{\mathbf{H}_{3}}(\alpha)=0$. Suppose that $\operatorname{var}(\Gamma) \subseteq \operatorname{var}(\alpha)$. Since $v_{\mathbf{H}_{3}}(\alpha)=0$ then, by Proposition 5 $v_{\mathbf{H}_{3}}(p) \in\{1,0\}$ for every propositional variable $p \in \operatorname{var}(\alpha)$. Thus $v_{\mathbf{H}_{3}}(\Gamma) \subseteq\{1\}$. Let $v_{\mathbf{C P L}}$ be a classical valuation such that $v_{\mathbf{C P L}}(p)=v_{\mathbf{H}_{3}}(p)$ for every $p \in \operatorname{var}(\alpha)=\operatorname{var}(\alpha) \cup \operatorname{var}(\Gamma)$. Then, by Proposition 6 , $v_{\mathbf{C P L}}(\Gamma) \subseteq\{1\}$ but $v_{\mathbf{C P L}}(\alpha)=0$, a contradiction. Then, $\operatorname{var}(\Gamma) \nsubseteq \operatorname{var}(\alpha)$. Thus, if $\operatorname{var}(\Gamma) \subseteq \operatorname{var}(\alpha)$ then $\Gamma \vDash_{\mathbf{H}_{3}} \alpha$.

Finally, if $\alpha$ is a classical tautology, let $v_{\mathbf{H}_{3}}$ be a valuation for $\mathbf{H}_{3}$. If $v_{\mathbf{H}_{3}}(p)=\frac{1}{2}$ for some $p \in \operatorname{var}(\alpha)$ then $v_{\mathbf{H}_{3}}(\alpha)=\frac{1}{2}$, by Proposition 5 . On the other hand, if $v_{\mathbf{H}_{3}}(\operatorname{var}(\alpha)) \subseteq\{0,1\}$ then, by Proposition 6 , $v_{\mathbf{H}_{3}}(\alpha)=1$. Then, $\vDash_{\mathbf{H}_{3}} \alpha$ and so $\Gamma \vDash_{\mathbf{H}_{3}} \alpha$.

So, by Theorem 7 we have that if a valid classical inference $\Gamma \vDash \alpha$ is invalid in Bochvar's nonsense logic then $\Gamma$ is a consistent set of formulas of CPL such that $\operatorname{var}(\alpha) \varsubsetneqq \operatorname{var}(\Gamma)$. On the other hand, Theorem 8 expresses that if a valid classical inference $\Gamma \vDash \alpha$ is invalid in Halldén's nonsense logic then $\alpha$ is not a tautological formula in CPL and $\operatorname{var}(\Gamma) \varsubsetneqq \operatorname{var}(\alpha)$. Therefore, it is clear that $\models_{\mathbf{H}_{3}} \alpha$ but $\nvdash_{\mathbf{B}_{3}} \alpha$, for every $\alpha$ such that $\vDash_{\text {CPL }} \alpha$.

By Theorems 7 and 8 we obtain a sufficient condition in order to determine whether a valid classical inference is also valid in both $\mathbf{B}_{3}$ and $\mathbf{H}_{3}$.

Corollary 9 Let $\Gamma \cup\{\alpha\}$ be a set of formulas in For 0 such that $\Gamma \vDash_{\mathbf{C P L}} \alpha$. Then: 2

$$
\text { if } \operatorname{var}(\Gamma)=\operatorname{var}(\alpha) \text {, then } \Gamma \vDash_{\mathbf{B}_{3}} \alpha \text { and } \Gamma \vDash_{\mathbf{H}_{3}} \alpha \text {. }
$$

We will introduce cut-free sequent calculi for the $\{\neg, \vee\}$-fragment of $\mathbf{H}_{3}$ and for the $\{\neg, \wedge\}$-fragment of $\mathbf{B}_{3}$, where $\wedge$ and $\rightarrow(\vee$ and $\rightarrow$, respectively) are derived connectives. The strategy adopted is to modify the classical sequent rules for classical connectives by adding suitable provisos. As we shall see, the provisos are applied to symmetrical rules: in the fragment of Halldén's logic, the provisos apply to the introduction rules for conjunction, implication and negation on the left side of the sequent while, in the case of Bochvar's logic, the proviso applies to the introduction rules for disjunction, implication and negation on the the right side. This reflects the relationship between these logics and classical logic, as depicted in theorems 7 and 8

\section{Sequent calculus $\mathbf{H}$ for the $\{\neg, \vee\}$-fragment of Halldén's logic $\mathbf{H}_{3}$}

As suggested by Theorem 8 , certain proofs in $\mathbf{C}$ should be blocked in any sequent calculus for $\mathbf{H}_{3}$. We present now a cut-free sequent calculus $\mathbf{H}$ for the fragment of $\mathbf{H}_{3}$ over $\Sigma_{1}$ by adding provisos on the application of (classical) rules such that the construction of complex formulas in the antecedent of the sequents is blocked in some cases. By symmetry, a sequent calculus $\mathbf{B}$ for $\mathbf{B}_{3}$ will be also introduced by adding provisos on the application of (classical) rules such that the construction of complex formulas in the succedents of the sequents is blocked under certain circumstances.

\footnotetext{
${ }^{2}$ Obviously we are identifying here a primitive connective of $\Sigma_{0}$ with its abbreviation in $\Sigma_{i}$, for $i=1,2$.
} 
Definition 10 The sequent calculus $\boldsymbol{H}$ is obtained from the $\{\neg, \vee\}$-fragment of $\boldsymbol{C}$ by replacing the rule $\neg \Rightarrow$ by the following one:

$$
\neg^{H} \Rightarrow \frac{\Gamma \Rightarrow \Delta, \alpha}{\neg \alpha, \Gamma \Rightarrow \Delta} \quad \text { provided that } \operatorname{var}(\alpha) \subseteq \operatorname{var}(\Delta)
$$

Proposition 11 The following rules are derivable in $\boldsymbol{H}$ :

$$
\wedge^{H} \Rightarrow \frac{\alpha_{1}, \alpha_{2}, \Gamma \Rightarrow \Delta}{\alpha_{1} \wedge \alpha_{2}, \Gamma \Rightarrow \Delta} \quad \Rightarrow \wedge \frac{\Gamma \Rightarrow \Delta, \alpha_{1} \quad \Gamma \Rightarrow \Delta, \alpha_{2}}{\Gamma \Rightarrow \Delta, \alpha_{1} \wedge \alpha_{2}}
$$

with the following proviso: $\operatorname{var}\left(\left\{\alpha_{1}, \alpha_{2}\right\}\right) \subseteq \operatorname{var}(\Delta)$ in $\wedge^{H} \Rightarrow$.

Proof. Assume that $\operatorname{var}\left(\left\{\alpha_{1}, \alpha_{2}\right\}\right) \subseteq \operatorname{var}(\Delta)$. Then $\operatorname{var}\left(\neg \alpha_{1} \vee \neg \alpha_{2}\right) \subseteq \operatorname{var}(\Delta)$ and so the following derivation can be done in $\mathbf{H}$ :

$$
\begin{gathered}
\frac{\alpha_{1}, \alpha_{2}, \Gamma \Rightarrow \Delta}{\Gamma \Rightarrow \Delta, \neg \alpha_{1}, \neg \alpha_{2}} \quad(\text { by } \Rightarrow \neg) \\
\frac{\Gamma \Rightarrow \Delta, \neg \alpha_{1} \vee \neg \alpha_{2}}{\neg\left(\neg \alpha_{1} \vee \neg \alpha_{2}\right), \Gamma \Rightarrow \Delta} \quad(\text { by } \Rightarrow \vee) \\
\left(\text { by } \neg^{H} \Rightarrow\right)
\end{gathered}
$$

In order to obtain $\Rightarrow \wedge$, the following derivation can be done in $\mathbf{H}$ :

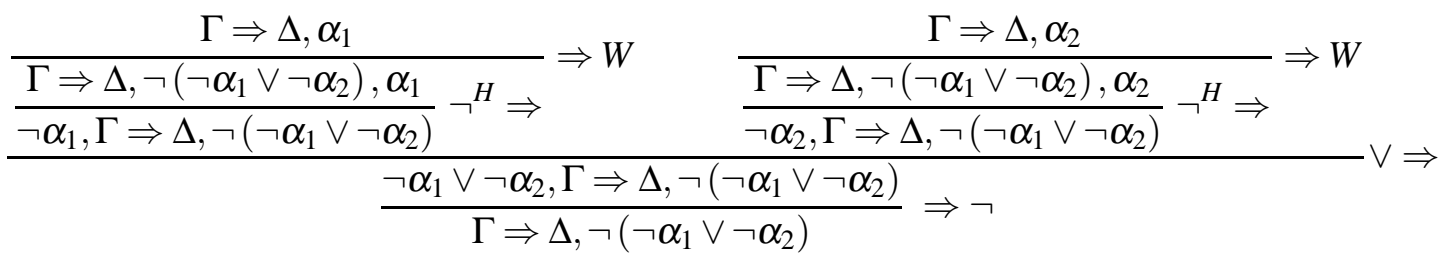

Proposition 12 The following implicational rules are derivable in $\boldsymbol{H}$ :

$$
\rightarrow^{H} \Rightarrow \frac{\Gamma \Rightarrow \Delta, \alpha_{1} \quad \alpha_{2}, \Gamma \Rightarrow \Delta}{\alpha_{1} \rightarrow \alpha_{2}, \Gamma \Rightarrow \Delta} \quad \Rightarrow \rightarrow \frac{\alpha_{1}, \Gamma \Rightarrow \Delta, \alpha_{2}}{\Gamma \Rightarrow \Delta, \alpha_{1} \rightarrow \alpha_{2}}
$$

with the following proviso: $\operatorname{var}\left(\left\{\alpha_{1}, \alpha_{2}\right\}\right) \subseteq \operatorname{var}(\Delta)$ in $\rightarrow^{H} \Rightarrow$.

Proof. Straightforward, by considering that $\alpha_{1} \rightarrow \alpha_{2}$ stands for $\neg \alpha_{1} \vee \alpha_{2}$ in $\mathbf{H}$.

\subsection{Soundness of $\mathbf{H}$}

In this subsection we shall prove the soundness of sequent calculus $\mathbf{H}$. Firstly, some semantical notions will be extended from formulas to sequents.

Definition 13 Let $\mathbf{L}$ be a matrix logic over a signature $\Sigma$. A valuation $v$ of $\boldsymbol{L}$ is a model of a sequent $\Gamma \Rightarrow \Delta$ over $\Sigma$ iff, if $v(\Gamma) \subseteq D_{\mathbf{L}}$, then $v(\delta) \in D_{\mathbf{L}}$ for some $\delta \in \Delta$. When $v$ is a model of the sequent $\Gamma \Rightarrow \Delta$, we will write $v \vDash_{\mathbf{L}} \Gamma \Rightarrow \Delta$. 
Definition $14 A$ sequent $\Gamma \Rightarrow \Delta$ is valid in $\boldsymbol{L}$ if, for every valuation $v$ of $\boldsymbol{L}, v$ is a model of the sequent $\Gamma \Rightarrow \Delta$. When the sequent is valid, we will write $\vDash_{\mathbf{L}} \Gamma \Rightarrow \Delta$.

It is worth noting that $\vDash_{\mathbf{L}} \Gamma \Rightarrow \alpha$ iff $\Gamma \vDash_{\mathbf{L}} \alpha$. Additionally, $\vDash_{\mathbf{C P L}} \Gamma \Rightarrow \Delta$ iff $\Gamma \vDash_{\mathbf{C P L}} \bigvee_{\alpha \in \Delta} \alpha$

Definition 15 A sequent rule $\mathfrak{R}$ preserves validity in $L$ if, for every instance $\frac{\Upsilon}{S}$ of $\mathfrak{R}$ and for every valuation $v$ of $\boldsymbol{L}$, if $v \vDash_{\mathbf{L}} S^{\prime}$ for every $S^{\prime} \in \Upsilon$ then $v \vDash_{\mathbf{L}} S$.

Lemma 16 Every sequent rule of the calculus $\boldsymbol{H}$ preserves validity.

Proof. Observe that the axiom Ax and the structural rules preserve validity, since they correspond to properties which are valid in every Tarskian logic (and $\mathbf{H}$ is Tarskian since it is a matrix logic).

$\Rightarrow \neg$ Let $v$ be a valuation of $\mathbf{H}_{3}$ such that $v \vDash_{\mathbf{H}_{3}} \alpha, \Gamma \Rightarrow \Delta$, and suppose that $v(\Gamma) \subseteq\left\{1, \frac{1}{2}\right\}$. If $v(\neg \alpha)=0$, then $v(\alpha)=1$. Then, by hypothesis, we infer that $v(\delta) \in\left\{1, \frac{1}{2}\right\}$, for some $\delta \in \Delta$. If $v(\neg \alpha) \neq 0$, then $v(\neg \alpha) \in\left\{1, \frac{1}{2}\right\}$. This shows that $v \vDash_{\mathbf{H}_{3}} \Gamma \Rightarrow \Delta, \neg \alpha$.

$\neg^{H} \Rightarrow$ Let $v$ be a valuation of $\mathbf{H}_{3}$ such that $v \vDash_{\mathbf{H}_{3}} \Gamma \Rightarrow \Delta, \alpha$ and assume that $\operatorname{var}(\alpha) \subseteq \operatorname{var}(\Delta)$. Suppose that $v(\neg \alpha) \in\left\{1, \frac{1}{2}\right\}$ and $v(\Gamma) \subseteq\left\{1, \frac{1}{2}\right\}$. Then, by hypothesis, $v(\delta) \in\left\{1, \frac{1}{2}\right\}$, for some $\delta \in \Delta$, or $v(\alpha) \in\left\{1, \frac{1}{2}\right\}$. Since $v(\neg \alpha) \in\left\{1, \frac{1}{2}\right\}$, then $v(\alpha) \in\left\{0, \frac{1}{2}\right\}$. If $v(\alpha)=0$ then $v(\delta) \in\left\{1, \frac{1}{2}\right\}$, for some $\delta \in \Delta$. And if $v(\alpha)=\frac{1}{2}$, then, by Proposition 5, we infer that $v(p)=\frac{1}{2}$ for some atomic formula $p \in \operatorname{var}(\alpha)$. Since $\operatorname{var}(\alpha) \subseteq \operatorname{var}(\Delta)$ then $p \in \operatorname{var}(\delta)$ for some $\delta \in \Delta$ and so, again by Proposition 5, we infer that $v(\delta)=\left\{\frac{1}{2}\right\}$. Therefore, we conclude that $v \vDash_{\mathbf{H}_{3}} \neg \alpha, \Gamma \Rightarrow \Delta$.

$\Rightarrow \vee$ Let $v$ be a valuation of $\mathbf{H}_{3}$ such that $v \vDash_{\mathbf{H}_{3}} \Gamma \Rightarrow \Delta, \alpha_{1}, \alpha_{2}$ and assume that $v(\Gamma) \subseteq\left\{1, \frac{1}{2}\right\}$. If $v\left(\alpha_{1}\right)=v\left(\alpha_{2}\right)=0$ then, by hypothesis, we infer that $v(\delta) \in\left\{1, \frac{1}{2}\right\}$, for some $\delta \in \Delta$. Therefore $v \vDash_{\mathbf{H}_{3}} \Gamma \Rightarrow \Delta, \alpha_{1} \vee \alpha_{2}$. Otherwise, if $v\left(\alpha_{1}\right) \in\left\{1, \frac{1}{2}\right\}$ or $v\left(\alpha_{2}\right) \in\left\{1, \frac{1}{2}\right\}$ then $v\left(\alpha_{1} \vee \alpha_{2}\right) \in\left\{1, \frac{1}{2}\right\}$ and so $v \vDash_{\mathbf{H}_{3}} \Gamma \Rightarrow \Delta, \alpha_{1} \vee \alpha_{2}$.

$\vee \Rightarrow$ Let $v$ be a valuation of $\mathbf{H}_{3}$ such that $v \vDash_{\mathbf{H}_{3}} \alpha_{1}, \Gamma \Rightarrow \Delta$ and $v \vDash_{\mathbf{H}_{3}} \alpha_{2}, \Gamma \Rightarrow \Delta$. Suppose that $v\left(\alpha_{1} \vee \alpha_{2}\right) \in$ $\left\{1, \frac{1}{2}\right\}$ and $v(\Gamma) \subseteq\left\{1, \frac{1}{2}\right\}$. Then, either $v\left(\alpha_{1}\right) \in\left\{1, \frac{1}{2}\right\}$ or $v\left(\alpha_{2}\right) \in\left\{1, \frac{1}{2}\right\}$. By hypothesis, it follows that $v(\delta) \in\left\{1, \frac{1}{2}\right\}$, for some $\delta \in \Delta$ and so $v \vDash_{\mathbf{H}_{3}} \alpha_{1} \vee \alpha_{2}, \Gamma \Rightarrow \Delta$.

Theorem 17 (Soundness of $\mathbf{H}$ ) Let $\Gamma \cup \Delta$ be a set of formulas in For ${ }_{1}$. Then: if $\Gamma \Rightarrow \Delta$ is provable in $\boldsymbol{H}$ then $\vDash_{\mathbf{H}_{3}} \Gamma \Rightarrow \Delta$. In particular, if $\Gamma \Rightarrow \alpha$ is provable in $\boldsymbol{H}$ then $\Gamma \vDash_{\mathbf{H}_{3}} \alpha$.

Proof. If the sequent $\Gamma \Rightarrow \Delta$ is an instance of axiom $\mathrm{Ax}$, then $\Gamma \Rightarrow \Delta$ is valid in $\mathbf{H}_{3}$. By induction on the depth of a derivation of $\Gamma \Rightarrow \Delta$ in $\mathbf{H}$ it follows, by the previous Lemma 16 , that the sequent $\Gamma \Rightarrow \Delta$ is valid in $\mathbf{H}_{3}$.

Proposition 18 Let $\Gamma$ be a nonempty set of formulas in For ${ }_{1}$. Then the sequent $\Gamma \Rightarrow$ is not provable in $\mathrm{H}$.

Proof. Let $v$ be a $\mathbf{H}_{3}$-valuation such that $v(p)=\frac{1}{2}$ for every $p \in \operatorname{var}(\Gamma)$. Then $v \nvdash_{\mathbf{H}_{3}} \Gamma \Rightarrow$ and so $\nvdash_{\mathbf{H}_{3}} \Gamma \Rightarrow$. By contraposition of Theorem 17 we conclude that the sequent $\Gamma \Rightarrow$ is not provable in $\mathbf{H}$. 


\subsection{Completeness of $\mathbf{H}$}

The following result follows straightforwardly:

Proposition 19 Let $\Gamma \cup \Delta$ be a finite nonempty set of formulas in For 1 . Then:

$$
\text { if } \vDash_{\mathbf{H}_{3}} \Gamma \Rightarrow \Delta \text {, then } \vDash_{\mathbf{C P L}} \Gamma \Rightarrow \Delta \text {. }
$$

Proof. Assume that $\models_{\mathbf{H}_{3}} \Gamma \Rightarrow \Delta$ and let $v$ be a classical valuation such that $v(\Gamma) \subseteq\{1\}$. By Proposition 6 , $v$ can be seen as a $\mathbf{H}_{3}$-valuation such that $v(\Gamma) \subseteq\left\{1, \frac{1}{2}\right\}$. By hypothesis, $v(\delta) \in\left\{1, \frac{1}{2}\right\}$ for some $\delta \in \Delta$. Since $v$ is classical, it follows that $v(\delta)=1$ for some $\delta \in \Delta$, therefore $\vDash_{\text {CPL }} \Gamma \Rightarrow \Delta$.

Proposition 20 Let $\Gamma \cup \Delta$ be a finite nonempty set of formulas in For ${ }_{1}$. Then: if $\Gamma \Rightarrow \Delta$ is provable in $\boldsymbol{H}$ then $\Gamma \Rightarrow \Delta$ is provable in the $\{\neg, \vee\}$-fragment of $\boldsymbol{C}$.

Proof. This is obvious, since $\mathbf{H}$ is a restricted version of the $\{\neg, \vee\}$-fragment of $\mathbf{C}$.

Lemma 21 Let $\Gamma \cup \Delta$ be a finite nonempty set of formulas in For 1 . Then: if $\Gamma \Rightarrow \Delta$ is provable in the $\{\neg, \vee\}$-fragment of $\boldsymbol{C}$ and $\operatorname{var}(\Gamma) \subseteq \operatorname{var}(\Delta)$ then $\Gamma \Rightarrow \Delta$ is provable in $\boldsymbol{H}$ without using the Cut rule.

Proof. Recall that derivations in $\mathbf{C}$ and $\mathbf{H}$ are rooted binary trees such that the root is the sequent being proved, and the leaves are always instances of the axiom Ax of the form $\alpha \Rightarrow \alpha$ for some formula $\alpha$.

Assume that $\Pi$ is a cut-free derivation in the $\{\neg, \vee\}$-fragment of $\mathbf{C}$ of a sequent $\Gamma \Rightarrow \Delta$ such that $\operatorname{var}(\Gamma) \subseteq \operatorname{var}(\Delta)$ (we can assume this by Theorem 4). If $\Pi$ is also a derivation in $\mathbf{H}$ then the result follows automatically. Otherwise, there are in $\Pi$, by force, applications of the rule $\neg \Rightarrow$, namely

$$
\neg \Rightarrow \frac{\Gamma^{\prime} \Rightarrow \Delta^{\prime}, \alpha}{\neg \alpha, \Gamma^{\prime} \Rightarrow \Delta^{\prime}}
$$

such that the proviso required by this rule in $\mathbf{H}$ is not satisfied. Since $\Pi$ is cut-free then the set of variables occurring in the root sequent $\Gamma \Rightarrow \Delta$ contains all the propositional variables occurring in $\Pi$. Then, by hypothesis, all the propositional variables occurring in $\Pi$ belong to the set $\operatorname{var}(\Delta)$. Consider now the derivation $\Pi^{\prime}$ in $\mathbf{C}$ obtained from $\Pi$ in two steps: firstly, the right-hand side of each sequent (that is, of each node) of $\Pi$ is enlarged by adding simultaneously all the formulas in $\Delta$. This generates a rooted binary tree $\Pi_{0}$ whose leafs are sequents of the form $\alpha \Rightarrow \alpha, \Delta$. Each of such leaves of $\Pi_{0}$ corresponds to the original occurrence of an axiom (that is, a leaf) $\alpha \Rightarrow \alpha$ in the derivation $\Pi$. In the second step, we replace each leaf $\alpha \Rightarrow \alpha, \Delta$ of $\Pi_{0}$ by a branch started by $\alpha \Rightarrow \alpha$ and followed by iterated applications of the weakening rule $\Rightarrow W$ until obtaining the sequent $\alpha \Rightarrow \alpha, \Delta$. The resulting rooted binary tree $\Pi^{\prime}$ is clearly a (cut-free) derivation in the $\{\neg, \vee\}$-fragment of $\mathbf{C}$ of the sequent $\Gamma \Rightarrow \Delta \sqrt{3}$ But the critical applications of the rule $\neg \Rightarrow$ mentioned above have in $\Pi^{\prime}$ the form

$$
\neg \Rightarrow \frac{\Gamma^{\prime} \Rightarrow \Delta^{\prime}, \Delta, \alpha}{\neg \alpha, \Gamma^{\prime} \Rightarrow \Delta^{\prime}, \Delta .}
$$

Being so, these applications are allowed in $\mathbf{H}$ (since all the propositional variables occurring in $\Pi^{\prime}$ belong to the set $\operatorname{var}(\Delta)$ ) and so $\Pi^{\prime}$ is in fact a cut-free derivation in $\mathbf{H}$ of the sequent $\Gamma \Rightarrow \Delta$. That is, $\Gamma \Rightarrow \Delta$ is provable in $\mathbf{H}$ without using the Cut rule.

Corollary 22 Let $\Delta$ be a finite nonempty set of formulas in For ${ }_{1}$. Then: $\Rightarrow \Delta$ is provable in the $\{\neg, \vee\}$ fragment of $\boldsymbol{C}$ if and only if $\Rightarrow \Delta$ is provable in $\boldsymbol{H}$.

\footnotetext{
${ }^{3}$ Observe that some applications of the weakening rule $\Rightarrow W$ in $\Pi$ may be innocuous in $\Pi^{\prime}$.
} 
Corollary 23 (Modus Ponens) Let $\alpha, \beta \in$ For $_{1}$. Then: if $\Rightarrow \alpha$ and $\Rightarrow \alpha \rightarrow \beta$ are provable in $\boldsymbol{H}$ then $\Rightarrow \beta$ is provable in $\boldsymbol{H}$.

Lemma 24 Let $\Gamma \cup \Delta$ be a finite nonempty set of formulas in For $_{1}$. If $\vDash_{\mathbf{H}_{3}} \Gamma \Rightarrow \Delta$ but var $(\Gamma) \varsubsetneqq \operatorname{var}(\Delta)$ then there exists $\Gamma^{\prime} \subset \Gamma$ such that $\models_{\mathbf{H}_{3}} \Gamma^{\prime} \Rightarrow \Delta$, where $\operatorname{var}\left(\Gamma^{\prime}\right) \subseteq \operatorname{var}(\Delta)$.

Proof. Observe that if $\vDash_{\mathbf{H}_{3}} \Gamma \Rightarrow \Delta$ then $\Delta \neq \emptyset$.

Assume that $\models_{\mathbf{H}_{3}} \Gamma \Rightarrow \Delta$ such that $\operatorname{var}(\Gamma) \varsubsetneqq \operatorname{var}(\Delta)$. So, given a valuation $v$ for $\mathbf{H}_{3}$, if $v(\Gamma) \subseteq\left\{1, \frac{1}{2}\right\}$ then $v(\delta) \in\left\{1, \frac{1}{2}\right\}$ for some formula $\delta \in \Delta$. Given that $\operatorname{var}(\Gamma) \varsubsetneqq \operatorname{var}(\Delta)$ consider the set $\Gamma^{\prime}=\Gamma \backslash$ $\{\gamma \in \Gamma: \operatorname{var}(\gamma) \varsubsetneqq \operatorname{var}(\Delta)\}$. Then, $\Gamma^{\prime} \subset \Gamma$ and $\operatorname{var}\left(\Gamma^{\prime}\right) \subseteq \operatorname{var}(\Delta)$. Let $v$ be a valuation for $\mathbf{H}_{3}$ such that $v\left(\Gamma^{\prime}\right) \subseteq\left\{1, \frac{1}{2}\right\}$. If $\frac{1}{2} \in v\left(\Gamma^{\prime}\right)$ then $v(p)=\frac{1}{2}$ for some propositional variable $p \in \operatorname{var}\left(\Gamma^{\prime}\right)$. Since $\operatorname{var}\left(\Gamma^{\prime}\right) \subseteq \operatorname{var}(\Delta)$, then $\frac{1}{2} \in v(\Delta)$. If $v\left(\Gamma^{\prime}\right) \subseteq\{1\}$, suppose that $v(\Delta)=\{0\}$. Then $v(p) \in\{1,0\}$ for every propositional variable $p \in \operatorname{var}(\Delta)$, by Proposition 5 . Since $\operatorname{var}\left(\Gamma^{\prime}\right) \subseteq \operatorname{var}(\Delta)$ then, for every propositional variable $p \in \operatorname{var}\left(\Gamma^{\prime}\right), v(p) \in\{1,0\}$. Consider now a valuation $v^{\prime}$ for $\mathbf{H}_{3}$ such that $v^{\prime}(p)=\frac{1}{2}$ for every $p \in \operatorname{var}(\Gamma) \backslash \operatorname{var}(\Delta)$, and $v^{\prime}(p)=v(p)$ for every $p \in \operatorname{var}(\Delta)$. Then, $v^{\prime}(\Gamma) \subseteq\left\{1, \frac{1}{2}\right\}$. But then, by hypothesis, $v^{\prime}(\delta) \in\left\{1, \frac{1}{2}\right\}$, for some $\delta \in \Delta$. That is, $v(\delta) \in\left\{1, \frac{1}{2}\right\}$ for some $\delta \in \Delta$, a contradiction. Therefore, if $v\left(\Gamma^{\prime}\right) \subseteq\{1\}$ then $v(\delta) \neq 0$, for some $\delta \in \Delta$. So, $\vDash_{\mathbf{H}_{3}} \Gamma^{\prime} \Rightarrow \Delta$.

Theorem 25 (Completeness of $\mathbf{H}$ ) Let $\Gamma \cup \Delta$ be a finite nonempty set of formulas in For ${ }_{1}$. If $\models_{\mathbf{H}_{3}} \Gamma \Rightarrow \Delta$ then $\Gamma \Rightarrow \Delta$ is provable in $\boldsymbol{H}$ without using the Cut rule. In particular, if $\Gamma \vDash_{\mathbf{H}_{3}} \alpha$ then the sequent $\Gamma \Rightarrow \alpha$ is provable in $\boldsymbol{H}$, for every finite set $\Gamma \cup\{\alpha\}$.

Proof. Assume that $\vDash_{\mathbf{H}_{3}} \Gamma \Rightarrow \Delta$. Then, by Proposition $19, \vDash_{\mathbf{C P L}} \Gamma \Rightarrow \Delta$. By Theorem $3, \Gamma \Rightarrow \Delta$ is provable in the $\{\neg, \vee\}$-fragment of $\mathbf{C}$. If $\operatorname{var}(\Gamma) \subseteq \operatorname{var}(\Delta)$ then, by Lemma $21, \Gamma \Rightarrow \Delta$ is provable in $\mathbf{H}$ without using the Cut rule. If $\operatorname{var}(\Gamma) \varsubsetneqq \operatorname{var}(\Delta)$ then, by Lemma 24, there exist a set $\Gamma^{\prime} \subset \Gamma$ such that $\models_{\mathbf{H}_{3}} \Gamma^{\prime} \Rightarrow \Delta$, where $\operatorname{var}\left(\Gamma^{\prime}\right) \subseteq \operatorname{var}(\Delta)$. Then, using Proposition 19 and Theorem 3 again, we obtain that $\Gamma^{\prime} \Rightarrow \Delta$ is provable in the $\{\neg, \vee\}$-fragment of $\mathbf{C}$. Since $\operatorname{var}\left(\Gamma^{\prime}\right) \subseteq \operatorname{var}(\Delta)$ then, by using Lemma 21, it follows that $\Gamma^{\prime} \Rightarrow \Delta$ is provable in $\mathbf{H}$ without using the Cut rule. By applying the structural rule $W \Rightarrow$ several times we obtain a derivation of $\Gamma \Rightarrow \Delta$ in $\mathbf{H}$ without using the Cut rule, as desired.

Corollary 26 (Cut elimination for $\mathbf{H}$ ) Let $\Gamma \cup \Delta$ be a finite nonempty set of formulas in For. If the sequent $\Gamma \Rightarrow \Delta$ is provable in $\boldsymbol{H}$ then there is a cut-free derivation of it in $\boldsymbol{H}$.

Proof. Suppose that $\Gamma \Rightarrow \Delta$ is provable in $\mathbf{H}$. By Theorem 17 $\vDash_{\mathbf{H}_{3}} \Gamma \Rightarrow \Delta$. Then, by Theorem 25, there is a cut-free derivation of $\Gamma \Rightarrow \Delta$ in $\mathbf{H}$.

\section{Sequent calculus $B$ for the $\{\neg, \wedge\}$-fragment of Bochvar's logic $B_{3}$}

In this section we introduce the sequent calculus $\mathbf{B}$ which will result cut-free, sound and complete for the conjunction-negation fragment of the nonsense logic $\mathbf{B}_{3}$, where $\vee$ and $\rightarrow$ are derived connectives. As we shall see, there exists a symmetry between the provisos imposed in the rules of $\mathbf{B}$ and those imposed in $\mathbf{H}$, as long as the language $\neg, \wedge, \vee, \rightarrow$ is considered.

Definition 27 The sequent calculus $\boldsymbol{B}$ is obtained from the $\{\neg, \wedge\}$-fragment of $\boldsymbol{C}$ by replacing the rule $\Rightarrow \neg$ by the following one:

$$
\Rightarrow \neg^{B} \frac{\alpha, \Gamma \Rightarrow \Delta}{\Gamma \Rightarrow \Delta, \neg \alpha} \quad \text { provided that } \operatorname{var}(\alpha) \subseteq \operatorname{var}(\Gamma)
$$


Proposition 28 The following rules are derivable in $\boldsymbol{B}$ :

$$
\vee \Rightarrow \frac{\alpha_{1}, \Gamma \Rightarrow \Delta \quad \alpha_{2}, \Gamma \Rightarrow \Delta}{\alpha_{1} \vee \alpha_{2}, \Gamma \Rightarrow \Delta} \quad \Rightarrow \vee^{B} \frac{\Gamma \Rightarrow \Delta, \alpha_{1}, \alpha_{2}}{\Gamma \Rightarrow \Delta, \alpha_{1} \vee \alpha_{2}}
$$

with the following proviso: $\operatorname{var}\left(\left\{\alpha_{1}, \alpha_{2}\right\}\right) \subseteq \operatorname{var}(\Gamma)$ in $\Rightarrow \vee^{B}$.

Proof. We leave the easy proof as an exercise to the reader.

Proposition 29 The following implicational rules are derivable in $\boldsymbol{B}$ :

$$
\rightarrow \frac{\Gamma \Rightarrow \Delta, \alpha_{1} \quad \alpha_{2}, \Gamma \Rightarrow \Delta}{\alpha_{1} \rightarrow \alpha_{2}, \Gamma \Rightarrow \Delta} \quad \Rightarrow \rightarrow^{B} \frac{\alpha_{1}, \Gamma \Rightarrow \Delta, \alpha_{2}}{\Gamma \Rightarrow \Delta, \alpha_{1} \rightarrow \alpha_{2}}
$$

with the following proviso: $\operatorname{var}\left(\left\{\alpha_{1}, \alpha_{2}\right\}\right) \subseteq \operatorname{var}(\Gamma)$ in $\Rightarrow \rightarrow^{B}$.

Proof. The proof is also left to the reader.

\subsection{Soundness of $B$}

In order to prove the Soundness Theorem for $\mathbf{B}$, we will prove that every sequent rule of the calculus $\mathbf{B}$ preserves validity.

Lemma 30 Every sequent rule of the calculus $\boldsymbol{B}$ preserves validity.

Proof. As in the case of $\mathbf{H}$, it is enough to analyze the rules for connectives.

$\Rightarrow \neg^{B}$ Assume that $v \models_{\mathbf{B}_{3}} \alpha, \Gamma \Rightarrow \Delta$ for some valuation $v$ in $\mathbf{B}_{3}$, where $\operatorname{var}(\alpha) \subseteq \operatorname{var}(\Gamma)$. Suppose that $v(\Gamma) \subseteq\{1\}$. Then, by Proposition $5, v(p) \in\{1,0\}$, for every propositional variable $p$ such that $p \in$ $\operatorname{var}(\Gamma)$. Since $\operatorname{var}(\alpha) \subseteq \operatorname{var}(\Gamma)$, then $v(p) \in\{1,0\}$, for every propositional variable $p \in \operatorname{var}(\alpha)$. By Proposition 5 again, we obtain that $v(\alpha) \in\{1,0\}$. If $v(\alpha)=1$, then by hypothesis, we obtain that $\{1\} \subseteq v(\Delta)$. If $v(\alpha)=0$ then $v(\neg \alpha)=1$. In both cases it follows that $\{1\} \subseteq v(\Delta \cup\{\neg \alpha\})$. Therefore $v \models_{\mathbf{B}_{3}} \Gamma \Rightarrow \Delta, \neg \alpha$.

$\neg \Rightarrow$ Assume that $v \models_{\mathbf{B}_{3}} \Gamma \Rightarrow \Delta, \alpha$ for some valuation $v$ in $\mathbf{B}_{3}$. Suppose that $v(\neg \alpha)=1$ and $v(\Gamma) \subseteq\{1\}$. So, $\{1\} \subseteq v(\Delta)$ or $v(\alpha)=1$, by hypothesis. But, since $v(\neg \alpha)=1$, then $v(\alpha)=0$. Thus, $\{1\} \subseteq$ $v(\Delta)$ and so $v \models_{\mathbf{B}_{3}} \neg \alpha, \Gamma \Rightarrow \Delta$.

$\Rightarrow \wedge$ Assume that $v \models_{\mathbf{B}_{3}} \Gamma \Rightarrow \Delta, \alpha_{1}$ and $v \models_{\mathbf{B}_{3}} \Gamma \Rightarrow \Delta, \alpha_{2}$ for some valuation $v$ in $\mathbf{B}_{3}$. Suppose that $v(\Gamma) \subseteq\{1\}$. By hypothesis, we obtain that either $\{1\} \subseteq v(\Delta)$ or both $v\left(\alpha_{1}\right)=1$ and $v\left(\alpha_{2}\right)=1$. In both cases it follows that $\{1\} \subseteq v\left(\Delta \cup\left\{\left(\alpha_{1} \wedge \alpha_{2}\right)\right\}\right)$. Then $v \models_{\mathbf{B}_{3}} \Gamma \Rightarrow \Delta, \alpha_{1} \wedge \alpha_{2}$.

$\wedge \Rightarrow$ Assume that $v \models_{\mathbf{B}_{3}} \alpha_{1}, \alpha_{2}, \Gamma \Rightarrow \Delta$ for some valuation $v$ in $\mathbf{B}_{3}$. Suppose that $v\left(\alpha_{1} \wedge \alpha_{2}\right)=1$ and $v(\Gamma) \subseteq\{1\}$. So, $v\left(\alpha_{1}\right)=v\left(\alpha_{2}\right)=1$ and $v(\Gamma) \subseteq\{1\}$. By hypothesis, $\{1\} \subseteq v(\Delta)$. Therefore, $v \models_{\mathbf{B}_{3}} \alpha_{1} \wedge \alpha_{2}, \Gamma \Rightarrow \Delta$.

As a consequence of this it follows the soundness theorem for $\mathbf{B}$ :

Theorem 31 (Soundness of B) Let $\Gamma \cup \Delta$ be a finite nonempty subset of For F $_{2}$ Then: if $\Gamma \Rightarrow \Delta$ is provable in $\boldsymbol{B}$ then $\models_{\mathbf{B}_{3}} \Gamma \Rightarrow \Delta$. In particular, if $\Gamma \Rightarrow \alpha$ is provable in $\boldsymbol{B}$ then $\Gamma \models \mathbf{B}_{3} \alpha$.

Corollary 32 Let $\Delta \subseteq$ For $_{2}$ be a nonempty set of formulas. Then the sequent $\Rightarrow \Delta$ is not provable in $\boldsymbol{B}$.

Proof. Consider a valuation $v$ for $\mathbf{B}_{3}$ such that $v(p)=\frac{1}{2}$ for every $p \in \operatorname{var}(\Delta)$. Then $v \forall_{\mathbf{B}_{3}} \Rightarrow \Delta$ and so $\forall \mathbf{B}_{3} \Rightarrow \Delta$. By Theorem 31 , the sequent $\Rightarrow \Delta$ is not provable in $\mathbf{B}$. 


\subsection{Completeness of $B$}

The proof of completeness of $\mathbf{B}$ is similar to that of $\mathbf{H}$ and so we will omit some proofs.

Proposition 33 Let $\Gamma \cup \Delta$ be a finite nonempty subset of For 2 . Then:

$$
\text { if } \models_{\mathbf{B}_{3}} \Gamma \Rightarrow \Delta \text {, then } \models \text { CPL } \Gamma \Rightarrow \Delta \text {. }
$$

Proposition 34 Let $\Gamma \cup \Delta$ be a finite nonempty subset of For 2 . Then: if $\Gamma \Rightarrow \Delta$ is provable in $\boldsymbol{B}$ then it is provable in the $\{\neg, \wedge\}$-fragment of $\boldsymbol{C}$.

Lemma 35 Let $\Gamma \cup \Delta$ be a finite nonempty subset of For. Then: if $\Gamma \Rightarrow \Delta$ is provable in the $\{\neg, \wedge\}$ fragment of $\boldsymbol{C}$ and $\operatorname{var}(\Delta) \subseteq \operatorname{var}(\Gamma)$ then $\Gamma \Rightarrow \Delta$ is provable in $\boldsymbol{B}$ without using the Cut rule.

Proof. The proof is analogous to that of Lemma 21, but now using the rule $W \Rightarrow$.

Lemma 36 Let $\Gamma \cup \Delta$ be a finite nonempty subset of $F_{0 r}$. If $\models_{\mathbf{B}_{3}} \Gamma \Rightarrow \Delta$ but var $(\Delta) \varsubsetneqq \operatorname{var}(\Gamma)$ then there exist a set $\Delta^{\prime} \subset \Delta$ such that $\models_{\mathbf{B}_{3}} \Gamma \Rightarrow \Delta^{\prime}$, where $\operatorname{var}\left(\Delta^{\prime}\right) \subseteq \operatorname{var}(\Gamma)$.

Proof. Let $\Delta^{\prime}=\Delta \backslash\{\delta \in \Delta: \operatorname{var}(\delta) \varsubsetneqq \operatorname{var}(\Gamma)\}$. Suppose that there is a $\mathbf{B}_{3}$-valuation $v$ such that $v(\Gamma) \subseteq$ $\{1\}$ but $v\left(\Delta^{\prime}\right) \subseteq\left\{0, \frac{1}{2}\right\}$. Thus, the $\mathbf{B}_{3}$-valuation $v^{\prime}$ such that $v^{\prime}(p)=v(p)$ for every $p \in \operatorname{var}(\Gamma)$ and $v^{\prime}\left(p^{\prime}\right)=\frac{1}{2}$ for every $p^{\prime} \in \operatorname{var}(\Delta) \backslash \operatorname{var}(\Gamma)$ is such that $v^{\prime}(\Gamma) \subseteq\{1\}$ but $v^{\prime}(\Delta) \subseteq\left\{0, \frac{1}{2}\right\}$, a contradiction. Therefore $\models_{\mathbf{B}_{3}} \Gamma \Rightarrow \Delta^{\prime}$, where $\operatorname{var}\left(\Delta^{\prime}\right) \subseteq \operatorname{var}(\Gamma)$.

Theorem 37 (Completeness of B) Let $\Gamma \cup \Delta$ be a finite nonempty subset of For $_{2}$. If $\models_{\mathbf{B}_{3}} \Gamma \Rightarrow \Delta$ then $\Gamma \Rightarrow \Delta$ is provable in $\boldsymbol{B}$ without using the Cut rule. In particular, if $\Gamma \models_{\mathbf{B}_{3}} \alpha$ then $\Gamma \Rightarrow \alpha$ is provable in $\boldsymbol{B}$.

Proof. Assume that $\models_{\mathbf{B}_{3}} \Gamma \Rightarrow \Delta$. Then, by Proposition 33 and Theorem 3, it follows that $\Gamma \Rightarrow \Delta$ is provable in the $\{\neg, \wedge\}$-fragment of $\mathbf{C}$. If $\operatorname{var}(\Delta) \subseteq \operatorname{var}(\Gamma)$ then, by Lemma 35, the sequent $\Gamma \Rightarrow \Delta$ is provable in $\mathbf{B}$ without using the Cut rule. On the other hand, if $\operatorname{var}(\Delta) \varsubsetneqq \operatorname{var}(\Gamma)$, then by Lemma 36 , $\vDash_{\mathbf{B}_{3}} \Gamma \Rightarrow \Delta^{\prime}$, for some set $\Delta^{\prime} \subset \Delta$ such that $\operatorname{var}\left(\Delta^{\prime}\right) \subseteq \operatorname{var}(\Gamma)$. By Proposition 33 and Theorem 3 again, it follows that $\Gamma \Rightarrow \Delta^{\prime}$ is provable in the $\{\neg, \wedge\}$-fragment of $\mathbf{C}$. Using again Lemma 35, the sequent $\Gamma \Rightarrow \Delta^{\prime}$ is provable in $\mathbf{B}$ without using the Cut rule. Finally, by applying the structural rule $\Rightarrow W$ several times we obtain a derivation of $\Gamma \Rightarrow \Delta$ in $\mathbf{B}$ without using the Cut rule.

Corollary 38 (Cut elimination for B) Let $\Gamma \cup \Delta$ be a finite nonempty set of formulas in For 2 . If the sequent $\Gamma \Rightarrow \Delta$ is provable in $\boldsymbol{B}$ then there is a cut-free derivation of it in $\boldsymbol{B}$.

Proof. Suppose that $\Gamma \Rightarrow \Delta$ is provable in $\mathbf{B}$. By Theorem 31 $\models_{\mathbf{B}_{3}} \Gamma \Rightarrow \Delta$. Then, by Theorem 37 there is a cut-free derivation of $\Gamma \Rightarrow \Delta$ in $\mathbf{B}$ as desired.

\section{Concluding Remarks}

In this paper a cut-free sequent calculi for the $\{\neg, \vee\}$-fragment of Bochvar's logic, as well as a cutfree sequent calculi for the $\{\neg, \wedge\}$-fragment of Halldén's logic, were proposed. In the former calculus, conjunction and implication are derived connectives, while disjunction and implication are derived connectives in the latter. The main feature of both calculi is that they are obtained by imposing provisos to the rules of the respective fragments of a well-known sequent calculus for classical propositional logic. The signature for each calculus was choosen in order to keep as close as possible to the respective fragment of classical logic. Observe that both $\{\neg, \vee\}$ and $\{\neg, \wedge\}$-fragments are adequate, that is, they can express all the other (classical) connectives. 
Thus, concerning the calculus for the $\{\neg, \vee\}$-fragment of Halldén's logic, the only change required with respect to the calculus for the respective fragment of classical logic was the inclusion of a proviso in the introduction rule for negation on the left side of the sequent. As a consequence of this, a proviso appear in the (derived) introduction rules for conjunction and implication on the left side of the sequent.

In the calculus for the $\{\neg, \wedge\}$-fragment of Bochvar's logic, the situation is entirely symmetrical: the restriction was imposed to the introduction rule for negation on the right side, and so this restriction also applies to the introduction rules for disjunction and implication on the right side (both are derived rules). In this manner, the existing relationship between classical logic and both logics became explicit through restrictions on the rules for the logical connectives.

Since these two logic of nonsense are related to classical logic in such particular way, the ad hoc definition of sequent calculi presented here, which exploit these particularities, seems to be justified. However, it would be interesting to compare the cut-free sequent calculi introduced here with the ones which could be obtained by applying general techniques such as those proposed in [2, 1, 8].

As a future research, we plan to extend the calculi to the full language of both logics. Clearly the resulting calculi will not be so simple and symmetrical because of the subtleties of the 'meaningful' connectives and their relationship with the other connectives.

Acknowledgements: We would like to thank the anonymous referees for their extremely useful comments on an earlier draft, which have helped to improve the paper. The first author was financed by FAPESP (Brazil), Thematic Project LogCons 2010/51038-0 and by an individual research grant from The National Council for Scientific and Technological Development (CNPq), Brazil.

\section{References}

[1] A. Avron, J. Ben-Naim \& B. Konikowska (2007): Cut-free Ordinary Sequent Calculi for Logics Having Generalized Finite-Valued Semantics. Logica Universalis 1(1), pp. 41-70, doi:10.1007/978-3-642-32621-9_ 24.

[2] M. Baaz, C.G. Fermüller \& R. Zach (1993): Systematic Construction of Natural Deduction Systems for Manyvalued Logics: Extended Report. Technical Report TUW-E185.2-BFZ.1-93.

[3] D.A. Bochvar (1938): Ob odnom trechzna čnom isčislenii i ego primenenii k analizu paradoksov klassiceskogo funkcional'nogo isčislenija. Matematicheskii Sbornik 4(46)(2), pp. 287-308. Translated to English by M. Bergmann as "On a Three-valued Logical Calculus and Its Application to the Analysis of the Paradoxes of the Classical Extended Functional Calculus". History and Philosophy of Logic 2:87-112, 1981.

[4] M.E. Coniglio \& M.I. Corbalán (2011): Recovering sense in the logics of nonsense (Recuperando o sentido nas lógicas do sem-sentido, in Portuguese). In: 16th Brazilian Logic Conference (XVI EBL): Book of Abstracts, Petrópolis, Brazil, pp. 38-38.

[5] M.I. Corbalán (2012): Local Recovering Connectives (Conectivos de Restauração Local, in Portuguese). Masters thesis, IFCH-State University of Campinas, Brazil.

[6] S. Halldén (1949): The Logic of Nonsense. Uppsala Univ., Uppsala.

[7] G. Malinowski (2007): Many-valued logic and its philosophy. In D.M. Gabbay \& J. Woods, editors: Handbook of the History of Logic, vol. 8: The Many Valued and Nonmonotonic Turn in Logic, North Holland, Amsterdam, pp. 13-94, doi:10.1016/S1874-5857(07)80004-5.

[8] M. Volpe, J. Marcos \& C. Caleiro (2012): Classic-Like Cut-Based Tableau Systems for Finite-Valued Logics. In: Proceedings of 19th WoLLIC, LNCS 7456, Springer, pp. 321-335, doi:10.1007/s11787-006-0003-6.

[9] T. Williamson (1994): Vagueness. Routledge, London \& New York. 